\title{
DYNAMICS VIA MEASURABILITY
}

\author{
SVETLANA BUTLER AND JOSEPH ROSENBLATT
}

Received 4 February 2005; Accepted 21 March 2005

Generators $f$ for $\sigma$-algebras can be used to view the dynamics of an invertible measurable transformation $T$ in terms of the range values of $f \circ T$. Such generators are the norm rather than the exception. Related measurable and quantitative methods of estimating a function from the behavior of ergodic averages are also discussed.

Copyright (c) 2006 S. Butler and J. Rosenblatt. This is an open access article distributed under the Creative Commons Attribution License, which permits unrestricted use, distribution, and reproduction in any medium, provided the original work is properly cited.

\section{Introduction}

There are many aspects of the dynamics of a measure-preserving transformation $T$, and the behavior of $f \circ T^{n}$ for some measurable function $f$, that can be understood in a revealing fashion by considering the $\sigma$-algebras that are generated by taking the smallest $\sigma$-algebra with respect to which the iterates $f \circ T, \ldots, f \circ T^{n}$ are measurable. In order to understand this in detail, some basic definitions and facts about measurability are needed. These facts are put in the second section. Then in the third section we discuss some properties of functions that are generic. In the fourth section, we discuss dynamics from the viewpoint of suitable measurable changes of variable in the range of functions and how this is related to various qualitative aspects of the dynamics of $T$. In the fifth section, we consider some quantitative estimates that arise from the ergodic averages, and how they may or may not give information about $f$, in contrast to what can be said from the method in the fourth section of this article.

\section{Structure of $\sigma$-algebras}

Let $(X, \Lambda, m)$ be a probability space. We do not always need more than this in the sequel, but sometimes it will be necessary to assume that the probability space is non-atomic and separable. So for simplicity, we assume throughout this article that $(X, \Lambda, m)$ is the closed interval $[0,1]$ with $m$ being the Lebesgue measure and $\Lambda$ being the Lebesgue measurable sets. In this context, denoting the Borel sets by $\mathbb{B}$, for any set $E \in \Lambda$ there exists $B \in \mathbb{B}$ 
such that $m(E \triangle B)=0$. We will take the usual convention of writing $E=B$ a.e. in such a situation, or when the null set by which they differ does not matter, just $E=B$. The symbol $\sqcup$ will denote the disjoint union of sets. Sets in $\mathbb{R}$ of the form $S=\bigsqcup_{i=1}^{n}\left(\alpha_{i}, \beta_{i}\right)$ will be called basic.

By a dynamical system we will mean $(X, \Lambda, m, T)$ where $T$ is an invertible bimeasurable transformation that preserves the measure $m$. An ergodic dynamical system will be a dynamical system in which $T$ is ergodic, that is, $T$ leaves invariant only the constant functions in $L_{1}(X)$. It will be convenient here to assume that all $\sigma$-algebras $\beta$ of sets in $\Lambda$ are completed with respect to null sets, that is, we take it that for any $A$ such that there exists $B \in \beta$ with $m(A \triangle B)=0$, we also have $A \in \beta$. The pseudo-metric $\rho$ is defined on $\Lambda$ by $\rho(A, B)=m(A \triangle B)$ for all $A, B \in \Lambda$. It is important that $\Lambda$ is a complete, pseudo-metric space with respect to $\rho$.

Given two sub- $\sigma$-algebras $\mathscr{B}_{1}$ and $\mathscr{B}_{2}$ of $\Lambda$, we are interested in simple criteria that tell us when $\mathscr{B}_{2} \subset \mathscr{B}_{1}$. Given $\mathscr{F}$, a set of $\Lambda$-measurable functions on $X$, we let $\sigma(\mathscr{F})$ denote the smallest sub- $\sigma$-algebra $\mathscr{B} \subset \Lambda$ such that all $f \in \mathscr{F}_{\mathscr{F}}$ are $\mathscr{B}$-measurable. We say that $\mathscr{F}_{\mathscr{F}}$ generates $\mathscr{B}$ if $\mathscr{B}=\sigma(\mathscr{F})$. All $\sigma$-algebras are of this form. Indeed, $\mathscr{B}=\sigma(\mathscr{F})$ if $\mathscr{F}=\left\{1_{E}\right.$ : $E \in \mathscr{B}\}$. We say that $\mathscr{B}$ is countably generated if there exists a countable set $\mathscr{F}$ such that $\mathscr{B}=\sigma(\mathscr{F})$. Thus, given two sets of $\Lambda$-measurable functions $\mathscr{F}$ and $\mathscr{G}$, we are generally interested in having simple criteria that would characterize when $\sigma(\mathscr{G}) \subset \sigma(\mathscr{F})$.

The results stated in this section may be well-known, but we could not find a good reference for them and so we have reproduced the short proofs so that the reader may understand the arguments without difficulty. The first fact is clear from the definitions.

Proposition 2.1. Suppose $\mathscr{F}$ is a set of $\Lambda$-measurable, real-valued functions on $X$. Then $\sigma(\mathscr{F})$ is the smallest sub- $\sigma$-algebra of $\Lambda$ that contains all $\sigma(f)$ for $f \in \mathscr{F}$.

Lemma 2.2. Suppose $f$ is a $\Lambda$-measurable, real valued function on $X$. Then with $\mathbb{B}$ being the Borel sets in $\mathbb{R}, \sigma(f)=f^{-1}(\mathbb{B})$.

Proof. Since $f$ is certainly $f^{-1}(\mathbb{B})$-measurable, we have $\sigma(f) \subset f^{-1}(\mathbb{B})$. On the other hand, consider the class $\tau$ of sets $E$ such that $f^{-1}(E) \in \sigma(f)$. Certainly, $\tau$ is a $\sigma$-algebra containing all the open sets. Thus, $\tau$ also must contain all the Borel sets. That is, $f^{-1}(\mathbb{B}) \subset$ $\sigma(f)$.

Lemma 2.3. A sub- $\sigma$-algebra $\mathscr{B}$ in $\Lambda$ is a closed set in the $\rho$-topology. Also, any sub- $\sigma$-algebra is countably generated.

Proof. Given a sequence $\left(A_{n}\right)$ in $\mathscr{B}$ which converges to $A$ in the $\rho$-topology, there is a subsequence $\left(A_{n_{m}}\right)$ such that $A=\bigcap_{M=1}^{\infty} \bigcup_{m=M}^{\infty} A_{n_{m}}$. Hence, the set $A \in \mathscr{B}$ too. So $\mathscr{B}$ is closed in the $\rho$-topology. In addition, since the $\rho$-topology is second countable, we see that $\mathscr{B}$ has a countable dense set too. It follows from the construction in the first part of the proof that a dense set in $\mathscr{B}$ necessarily generates $\mathscr{B}$. Hence, $\mathscr{B}$ is countably generated.

Corollary 2.4. For any sub- $\sigma$-algebra $\mathscr{B}$ in $\Lambda$, there exists a function $f \in L_{1}(X)$ such that $\sigma(f)=\mathscr{B}$.

Proof. By Lemma 2.3, there is a countable set $\left\{E_{n}: n \geq 1\right\}$ that generates $\mathscr{B}$. Let $f=$ $\sum_{n=1}^{\infty}\left(1 / 3^{n}\right) 1_{E_{n}}$. Clearly, $f$ is $\mathscr{B}$-measurable, so $\sigma(f) \subset \mathscr{B}$. Also, $\sigma(f)$ contains all the 
sets $E_{n}$. Indeed, for all $n \geq 1$, the set $E_{n}=f^{-1} 1 /\left(2 \cdot 3^{n}, 2 / 3^{n}\right)$. Hence, $\mathscr{B} \subset \sigma(f)$ because $\left\{E_{n}: n \geq 1\right\}$ generates $\mathscr{B}$.

Remark 2.5. We will say that $f$ is a generator for $\mathscr{B}$, a sub- $\sigma$-algebra of $\Lambda$, when $\sigma(f)=\mathscr{B}$. If $\mathscr{B}$ is actually equal to $\Lambda$, then we will just say that $f$ is a generator. Corollary 2.4 is saying that all sub- $\sigma$-algebras $\mathscr{B}$ in $\Lambda$ have generators.

Lemma 2.6. Suppose $f$ and $F$ are $\Lambda$-measurable, real-valued functions. Then $\sigma(f) \subset \sigma(F)$ if and only if there is a Borel measurable function h such that $f=h \circ F$ a.e.

Proof. Using Lemma 2.2 and the Borel measurability of $h$, the existence of $h$ implies that $\sigma(f)=\sigma(h \circ F)=(h \circ F)^{-1}(\mathbb{B})=F^{-1}\left(h^{-1}(\mathbb{B})\right) \subset F^{-1}(\mathbb{B})=\sigma(F)$. Conversely, suppose $\sigma(f) \subset \sigma(F)$. By partitioning the range of $f$, we can construct a sequence of $\sigma(f)$ measurable simple functions $\phi_{n}$ such that $\lim _{n \rightarrow \infty} \phi_{n}(x)=f(x)$ for all $x \in X$. Now each $\phi_{n}$ is a finite sum of the form $\sum_{i} c_{n}(i) 1_{E_{n}(i)}$ where each $E_{n}(i) \in \sigma(f)$. Since $F$ is $\Lambda$-measurable and $f$ is $\sigma(F)$-measurable, by Lemma 2.2 we have $\sigma(f) \subseteq \sigma(F)=F^{-1}(\mathscr{B})$, and we can choose Borel sets $D_{n}(i)$ such that for all $n$ and $i, E_{n}(i)=F^{-1}\left(D_{n}(i)\right)$ a.e. That is, for each $n$, we have $\phi_{n}=\sum_{i} c_{n}(i) 1_{D_{n}(i)} \circ F$ a.e. Let $h=\limsup _{n \rightarrow \infty} \sum_{i} c_{n}(i) 1_{D_{n}(i)}$. Then we have for a.e. $x \in X$,

$$
f(x)=\lim _{n \rightarrow \infty} \phi_{n}(x)=\limsup _{n \rightarrow \infty} \phi_{n}(x) .
$$

So we have

$$
f(x)=\limsup _{n \rightarrow \infty} \sum_{i} c_{n}(i) 1_{D_{n}(i)}(F(x))=h(F(x)) .
$$

Remark 2.7. (a) This lemma does not remain true if we allow $h$ to be just Lebesgue measurable. For example, let $C$ be the usual middle-thirds Cantor set. There is a one-to-one, onto, Borel measurable function $F: X \rightarrow C$ whose inverse is Borel measurable too. That is, $X$ and $C$ are Borel equivalent; see Royden [3, Chapter 15, Section 4]. Using a cardinality argument, there exists $E \subset C$ such that $F^{-1}(E)$ is not $\Lambda$-measurable. But then $h=1_{E}$ is $\Lambda$-measurable because $C$ is a null set. We have $(h \circ F)^{-1}(\{1\})=F^{-1}(E)$ is not in $\Lambda$. So if $f=h \circ F$, we cannot have $\sigma(f) \subset \sigma(F)$ because $\sigma(F) \subset \Lambda$, while $f^{-1}(\{1\}) \notin \Lambda$.

(b) It follows from Corollary 2.4 and Lemma 2.6 that any two generators $f_{1}$ and $f_{2}$ of a $\sigma$-algebra $\beta$ are equivalent in the sense that there exist Borel measurable functions $h_{1}$ and $h_{2}$ such that $h_{1} \circ f_{2}=f_{1}$ a.e. and $h_{2} \circ f_{1}=f_{2}$ a.e. If these identities held everywhere, with $f_{1}$ and $f_{2}$ being also surjective functions, then $h_{1}$ and $h_{2}$ are inverses of one another. But in general, because $f_{1}$ or $f_{2}$ might not preserve null sets, we cannot even conclude from these identifies that $h_{1}$ and $h_{2}$ are a.e. inverses of one another.

Proposition 2.8. Given a sequence $\left(f_{n}: n \geq 1\right)$ of $\Lambda$-measurable functions, there exists a Borel measurable $h$ such that

$$
\sigma\left(\left\{f_{n}: n \geq 1\right\}\right)=\sigma\left(h\left(f_{1}, f_{2}, f_{3}, \ldots\right)\right) .
$$

Before proving this result, here is an immediate corollary of it. 
Corollary 2.9. Given a sequence $\left(f_{n}: n \geq 1\right)$ of $\Lambda$-measurable functions, if $f$ is $\sigma\left(\left\{f_{n}: n \geq\right.\right.$ $1\})$-measurable, then there exists a Borel measurable $h$ such that $f=h\left(f_{1}, f_{2}, f_{3}, \ldots\right)$ a.e.

Proof. Using Proposition 2.8, we have $\sigma(f) \subset \sigma\left(h_{1}\left(f_{1}, f_{2}, f_{3}, \ldots\right)\right)$ for a suitable Borel measurable function $h_{1}$. Then by Lemma 2.6, there is another Borel measurable function $h_{2}$ such that $f=h_{2} \circ h_{1}\left(f_{1}, f_{2}, f_{3}, \ldots\right)$ a.e. So $h=h_{2} \circ h_{1}$ gives this corollary.

To prove Proposition 2.8 it is useful to separate out the following lemma.

Lemma 2.10. Given a sequence $\left(f_{n}: n \geq 1\right)$ of $\Lambda$-measurable functions, and a Borel measurable function $h: \mathbb{R}^{\infty} \rightarrow \mathbb{R}, h\left(f_{1}, f_{2}, f_{3}, \ldots\right)$ is $\sigma\left(\left\{f_{n}: n \geq 1\right\}\right)$-measurable.

Proof. The Borel sets in $\mathbb{R}^{\infty}$ form a $\sigma$-algebra that is generated by open sets of the form $\prod_{k=1}^{\infty} U_{k}$ where each $U_{k}$ is open in $\mathbb{R}$, and only finitely many of them are different from $\mathbb{R}$. Consider the sets $\Sigma=\left\{E \subset \mathbb{R}^{\infty}:\left(f_{1}, f_{2}, f_{3}, \ldots\right)^{-1}(E) \in \sigma\left(\left\{f_{1}, f_{2}, f_{3}, \ldots\right\}\right)\right\}$. This is clearly a $\sigma$-algebra. It also contains any set $E$ of the form $\prod_{k=1}^{\infty} U_{k}$ where each $U_{k}$ is open in $\mathbb{R}$, and only finitely many of them are different from $\mathbb{R}$. Hence, it contains the Borel sets in $\mathbb{R}^{\infty}$. But for any open set $U$ in $\mathbb{R}$, the set $h^{-1}(U)$ is a Borel set in $\mathbb{R}^{\infty}$. Hence, for any open set $U$ in $\mathbb{R}, h\left(f_{1}, f_{2}, f_{3}, \ldots\right)^{-1}(U)=\left(f_{1}, f_{2}, f_{3}, \ldots\right)^{-1} h^{-1}(U)$ is in $\sigma\left(\left\{f_{n}: n \geq 1\right\}\right)$. That is, $h\left(f_{1}, f_{2}, f_{3}, \ldots\right)$ is $\sigma\left(\left\{f_{n}: n \geq 1\right\}\right)$-measurable.

Proof of Proposition 2.8. By Lemma 2.10, we only need to find a Borel measurable function $h$ such that $\sigma\left(\left\{f_{n}: n \geq 1\right\}\right) \subset \sigma\left(h\left(f_{1}, f_{2}, f_{3}, \ldots\right)\right)$. It is well-known that there is a oneto-one, onto mapping $h: \mathbb{R}^{\infty} \rightarrow \mathbb{R}$ that is Borel measurable and whose inverse in Borel measurable. That is, $\mathbb{R}$ and $\mathbb{R}^{\infty}$ are Borel equivalent; see Royden [3, Chapter 15, Section 4]. This function $h$ will do what we want. Indeed, suppose $U \subset \mathbb{R}$ is open and fix $k \geq 1$. Let $U_{n}=\mathbb{R}$ for all $n \neq k$ and let $U_{k}=U$. Because $h$ is a Borel equivalence, there is a Borel measurable set $E$ such that $h^{-1}(E)=\prod_{n=1}^{\infty} U_{n}$. Hence, $f_{k}^{-1}(U)=h\left(f_{1}, f_{2}, f_{3}, \ldots\right)^{-1}(E)$. By Lemma $2.2, h\left(f_{1}, f_{2}, f_{3}, \ldots\right)^{-1}(E)$ is in $\sigma\left(h\left(f_{1}, f_{2}, f_{3}, \ldots\right)\right)$. Thus, for any open set $U$ and any fixed $k \geq 1$, the inverse image $f_{k}^{-1}(U) \in \sigma\left(h\left(f_{1}, f_{2}, f_{3}, \ldots\right)\right)$. Hence, for all $k \geq 1, \sigma\left(f_{k}\right) \subset$ $\sigma\left(h\left(f_{1}, f_{2}, f_{3}, \ldots\right)\right)$. That is, by Proposition 2.1, $\sigma\left(\left\{f_{k}: k \geq 1\right\}\right) \subset \sigma\left(h\left(f_{1}, f_{2}, f_{3}, \ldots\right)\right)$.

\section{Generators}

We have seen that there always are generators, even bounded Borel measurable functions $f$ such that $\Lambda=\sigma(f)$. Indeed, most functions are like this in the sense of Baire category. Here is a proof of one version of this statement.

Proposition 3.1. The generators are dense in $L_{1}(X)$.

Proof. Let $f_{0} \in L_{1}(X)$. For a given $\epsilon>0$, we want to find a generator $f \in L_{1}(X)$ such that $\left\|f-f_{0}\right\|_{1}<\epsilon$. Since $\Lambda$ is countably generated, we can choose countably many measurable sets $E_{1}, E_{2}, \ldots$ such that $\sigma\left(E_{1}, E_{2}, \ldots\right)=\Lambda$. Let $E_{0}=\left\{x:\left|f_{0}(x)\right| \geq \epsilon / 2\right\}$. Then $\| f_{0}-$ $f_{0} 1_{E_{0}} \|_{1}<\epsilon / 2$. Suppose $\sum_{n=1}^{\infty} a_{n}$ is a convergent series of positive terms with the sum less than $\epsilon / 2$. Consider

$$
f=f_{0} 1_{E_{0}}+\sum_{n=1}^{\infty} a_{n} 1_{E_{n}} .
$$


Then $f \in L_{1}(X)$ and $\left\|f-f_{0}\right\|_{1}<\epsilon$. But we can also arrange for each $E_{n}$ to be $f^{-1}\left(I_{n}\right)$ for a suitable open interval around $a_{n}$. Indeed, for this to happen, it suffices to take $\left(a_{n}\right)$ to be strictly decreasing and to have $a_{n}>\sum_{k=n+1}^{\infty} a_{k}$ for all $n \geq 1$. Then if $I_{n}=\left(\alpha_{n}, \beta_{n}\right)$ with $a_{n-1}>\alpha_{n}>a_{n}>\beta_{n}>\sum_{k=n+1}^{\infty} a_{k}$ we would know that $E_{n}=f^{-1}\left(I_{n}\right)$.

Given an interval $I$ with endpoints $\alpha$ and $\beta$, and given $\gamma>0$, we denote by $I_{\gamma}$ the inter$\operatorname{val}(\alpha+\gamma, \beta-\gamma)$.

Lemma 3.2. Let $f \in L_{1}(X)$. If $I=(\alpha, \beta)$ is an open interval and $\epsilon>0$, then there exists $\gamma_{0}>0$ such that for all $\gamma<\gamma_{0}$, the interval $I_{\gamma}$ has $m\left(f^{-1}(I) \triangle f^{-1}\left(I_{\gamma}\right)\right)<\epsilon$. If $I=[\alpha, \beta]$ is a closed interval and $\epsilon>0$, then there exists $\gamma_{0}>0$ such that for all $\gamma<\gamma_{0}$, the interval $I_{-\gamma}$ has $m\left(f^{-1}(I) \triangle f^{-1}\left(I_{\gamma}\right)\right)<\epsilon$.

Proof. The proofs are similar. In the case that $I$ is open, use the fact that $I=\bigcup_{k=1}^{\infty} I_{1 / k}$. Thus, taking inverse images and using the finiteness of $m$ finishes the argument. In the case that $I$ is closed, use the fact that $I=\bigcap_{k=1}^{\infty} I_{-1 / k}$. Then proceed similarly to the argument in the case when $I$ is open.

Remark 3.3. We will not only use the notation $I_{\gamma}$ above, but we also extend it to basic sets. If the basic set $S=\bigsqcup_{i=1}^{n}\left(\alpha_{i}, \beta_{i}\right)$, then $S_{\gamma}=\bigsqcup_{i=1}^{n}\left(\alpha_{i}+\gamma, \beta_{i}-\gamma\right)$.

Proposition 3.4. Suppose $f \in L_{1}(X)$. A measurable set $E \in \sigma(f)$ if and only if for any $\epsilon>0$, there exists a basic set $S$ such that

$$
m\left(f^{-1}(S) \triangle E\right)<\epsilon
$$

Proof. Consider $\tau$, the class of sets defined by $E \in \tau$ if and only if for any $\epsilon>0$ there exists a basic set $S$ such that $m\left(f^{-1}(S) \triangle E\right)<\epsilon$. We will show that $\tau=\sigma(f)$.

First, it is clear that $\tau$ is a $\sigma$-algebra. Indeed, first suppose $E \in \tau, \epsilon>0$ is given and $S=\bigsqcup_{i=1}^{n}\left(\alpha_{i}, \beta_{i}\right)$ is a basic set such that $m\left(f^{-1}(S) \triangle E\right)<\epsilon / 2$. Then the complement $S^{c}$ of $S$ is a finite union of disjoint closed sets and we may use Lemma 3.2 to find a basic set $S^{\prime}$ such that $m\left(f^{-1}\left(S^{c}\right) \triangle f^{-1}\left(S^{\prime}\right)\right)<\epsilon / 2$. Then

$$
\begin{aligned}
m\left(E^{c} \triangle f^{-1}\left(S^{\prime}\right)\right) & \leq m\left(E^{c} \triangle f^{-1}\left(S^{c}\right)\right)+m\left(f^{-1}\left(S^{c}\right) \triangle f^{-1}\left(S^{\prime}\right)\right) \\
& =m\left(E \triangle f^{-1}(S)\right)+m\left(f^{-1}\left(S^{c}\right) \triangle f^{-1}\left(S^{\prime}\right)\right) \\
& <\frac{\epsilon}{2}+\frac{\epsilon}{2}=\epsilon,
\end{aligned}
$$

which shows that $E^{c}$ in $\tau$. Also, suppose $E_{i} \in \tau$ for $i=1,2, \ldots$. We want to show that $E=\bigcup_{i=1}^{\infty} E_{i}$ is also in $\tau$. Let $\epsilon>0$. Choose basic sets $S_{i}, i=1,2, \ldots$ such that

$$
m\left(E_{i} \triangle f^{-1}\left(S_{i}\right)\right)<\frac{\epsilon}{2^{i+1}} .
$$

Let $M \in \mathbb{N}$ be such that for the set $U_{M}=\bigcup_{i=1}^{M} E_{i}$ we have $m\left(E \triangle U_{M}\right)<\epsilon / 2$. The open set $S=\bigcup_{i=1}^{M} S_{i}$ can be written as a finite disjoint union of open intervals. Hence, $S$ is a basic 
set and:

$$
\begin{aligned}
m\left(E \triangle f^{-1}(S)\right) & \leq m\left(E \triangle U_{M}\right)+m\left(U_{M} \triangle f^{-1}(S)\right) \\
& \leq \frac{\epsilon}{2}+\sum_{i=1}^{M} m\left(E_{i} \triangle f^{-1}\left(S_{i}\right)\right) \\
& <\frac{\epsilon}{2}+\frac{\epsilon}{2}=\epsilon .
\end{aligned}
$$

Thus, $\tau$ is a $\sigma$-algebra. But also every open interval $I$ is itself a basic set and so we have $f^{-1}(I) \in \tau$ by the definition of $\tau$. It follows that $\sigma(f) \subset \tau$.

Suppose that $E \in \tau$. For $i=1,2, \ldots$, pick a basic set $S_{i}$ in $\mathbb{R}$ such that $m\left(E \triangle f^{-1}\left(S_{i}\right)\right)<$ $1 / 2^{i}$. Let $A_{i}=f^{-1}\left(S_{i}\right)$ and $A=\bigcap_{k=1}^{\infty} \bigcup_{i=k}^{\infty} A_{i}$. Then each $A_{i} \in \sigma(f)$ and so $A \in \sigma(f)$. Moreover, it is not difficult to show that for any given $\epsilon>0$ we have $m(E \triangle A)<\epsilon$. It follows that $m(E \triangle A)=0$. As we stated in the introduction, all subalgebras of $\Lambda$ are completed with respect to null sets. So $E \in \sigma(f)$, hence, $\sigma(f)=\tau$.

Proposition 3.5. Let $f_{n} \rightarrow f$ in $L_{1}(X)$-norm. Suppose that $E \in \Lambda$ and $\delta \geq 0$ are such that for all $n \in \mathbb{N}$ and for all basic sets $S$ we have

$$
m\left(E \triangle f_{n}^{-1}(S)\right) \geq \delta
$$

It follows that for all basic sets $S$ we also have the inequality

$$
m\left(E \triangle f^{-1}(S)\right) \geq \delta
$$

Proof. Suppose that $S$, a basic set, and $\epsilon>0$ are given. We will use basic sets $S_{\gamma}$ and $S_{2 \gamma}$ defined as in Remark 3.3. We choose $S_{2 \gamma}$ so that

$$
m\left(f^{-1}(S) \triangle f^{-1}\left(S_{2 \gamma}\right)\right)<\epsilon
$$

Notice that

$$
f^{-1}\left(S_{2 \gamma}\right) \subset f^{-1}\left(S_{\gamma}\right)
$$

Since $f_{n} \rightarrow f$ in $L_{1}(X)$, by choosing an appropriate subsequence if necessary, we may assume that $f_{n} \rightarrow f$ a.e. on $X$. Then there exists $X^{\prime} \subset X$ with $m\left(X \backslash X^{\prime}\right)<\epsilon$ on which $f_{n}$ converges to $f$ uniformly. Notice that because of the uniform convergence on $X^{\prime}$, restricting to $X^{\prime}$ we can make uniform approximations. Hence, we have for large enough $n$ :

$$
\begin{gathered}
f^{-1}\left(S_{2 \gamma}\right) \cap X^{\prime} \subset f_{n}^{-1}\left(S_{\gamma}\right) \cap X^{\prime}, \\
f_{n}^{-1}\left(S_{\gamma}\right) \cap X^{\prime} \subset f^{-1}(S) \cap X^{\prime} .
\end{gathered}
$$


The last three inclusions imply that restricting to $X^{\prime}$ we have,

$$
\begin{aligned}
f^{-1}\left(S_{2 \gamma}\right) \triangle f_{n}^{-1}\left(S_{\gamma}\right) & =\left(f^{-1}\left(S_{2 \gamma}\right) \backslash f_{n}^{-1}\left(S_{\gamma}\right)\right) \sqcup\left(f_{n}^{-1}\left(S_{\gamma}\right) \backslash f^{-1}\left(S_{2 \gamma}\right)\right) \\
& \subset\left(f^{-1}(S) \backslash f_{n}^{-1}\left(S_{\gamma}\right)\right) \cup\left(f^{-1}(S) \backslash f^{-1}\left(S_{2 \gamma}\right)\right) \\
& \subset\left(f^{-1}(S) \backslash f^{-1}\left(S_{2 \gamma}\right)\right) \cup\left(f^{-1}(S) \backslash f^{-1}\left(S_{2 \gamma}\right)\right) \\
& =f^{-1}(S) \backslash f^{-1}\left(S_{2 \gamma}\right) .
\end{aligned}
$$

Then by (3.8) we know that

$$
m\left(\left(f^{-1}\left(S_{2 \gamma}\right) \triangle f_{n}^{-1}\left(S_{\gamma}\right)\right) \cap X^{\prime}\right) \leq m\left(\left(f^{-1}(S) \backslash f^{-1}\left(S_{2 \gamma}\right)\right) \cap X^{\prime}\right)<\epsilon .
$$

Hence we have:

$$
\begin{aligned}
\delta \leq & m\left(E \triangle f_{n}^{-1}\left(S_{\gamma}\right)\right) \\
\leq & m\left(E \triangle f^{-1}(S)\right)+m\left(f^{-1}(S) \triangle f^{-1}\left(S_{2 \gamma}\right)\right)+m\left(f^{-1}\left(S_{2 \gamma}\right) \triangle f_{n}^{-1}\left(S_{\gamma}\right)\right) \\
\leq & m\left(E \triangle f^{-1}(S)\right)+m\left(f^{-1}(S) \triangle f^{-1}\left(S_{2 \gamma}\right)\right) \\
& +m\left(f^{-1}\left(S_{2 \gamma}\right) \triangle f_{n}^{-1}\left(S_{\gamma}\right) \cap X^{\prime}\right)+m\left(X \backslash X^{\prime}\right) \\
\leq & m\left(E \triangle f^{-1}(S)\right)+\epsilon+\epsilon+\epsilon \\
= & m\left(E \triangle f^{-1}(S)\right)+3 \epsilon .
\end{aligned}
$$

Since $\epsilon$ is arbitrary, we have $m\left(E \triangle f^{-1}(S)\right) \geq \delta$.

The upshot of these propositions is the next theorem that shows that typically a function $f \in L_{1}(X)$ is a generator.

Theorem 3.6. The set of generators is residual.

Proof. For $E \in \Lambda$ and a positive $\delta$ consider

$$
D_{E, \delta}=\left\{f \in L_{1}(X): \text { for every basic set } S \subset \mathbb{R} m\left(E \triangle f^{-1}(S)\right) \geq \delta\right\} .
$$

Then each such set $D_{E, \delta}$ is closed in $L_{1}$-norm by Proposition 3.5. Each $D_{E, \delta}$ has empty interior by Propositions 3.1 and 3.4. Fix a countable set $Q$ which is dense in $\Lambda$ with respect to the topology given by pseudo-metric $\rho$. By Lemma 2.3, $\sigma(f)$ is closed and so $f$ is not a generator if and only if there exists $E \in Q$ such that $E \notin \sigma(f)$. Then by Proposition 3.4, we have $f$ is not generator if and only if $f \in \bigcup_{E \in Q} \bigcup_{k=1}^{\infty} D_{E, 1 / k}$. Thus, the set of functions that are not generators is meager and the set of functions that are generators is residual.

\section{Dynamics via measurability}

Consider first the situation where $f$ is a generator. Let $T$ be any measure-preserving transformation on $(X, \Lambda, m)$. We know that $\sigma(f \circ T)=\Lambda=\sigma(f)$. Hence, by Lemma 2.6, there exists a Borel measurable function $h$ such that $f \circ T=h \circ f$. But then $f \circ T^{2}=$ $(h \circ f) \circ T=h \circ h \circ f$. That is, inductively $f \circ T^{n}=h^{n}(f)$ for all $n \geq 1$. This means that generically any ergodic transformation has a dynamical behavior that can be viewed as just rearranging the range of the function it is acting on, instead of rearranging its domain 
as it obviously does. Also, there is a Borel measurable function $k$ such that $k(f \circ T)=f$. Hence, $k \circ h=I d$ on the range of $f$ and $h \circ k=I d$ on the range of $f$. So $f \circ T$ and $f$ are the same function up to a one-to-one, onto rearrangement of the range values. It is easy to see that generically, the generator $f$ also has a range that is all of $\mathbb{R}$, so the functions $h$ and $k$ are even Borel equivalences of $\mathbb{R}$ that are inverses of one another.

This discussion really does not explicitly use the fact that $T$ is measure-preserving. One really only needs an invertible bimeasurable point transformation $T: X \rightarrow X$. So take such a general mapping $T$ and assume that $f$ is a generator; then there exists a Borel measurable mapping $h$ such that $f \circ T=h \circ f$. How does one characterize $T$ being measurepreserving in terms of properties of $h$ ?

We can see a suggested characterization in terms of distribution functions. The distribution function of $f \in L_{1}(X)$ is denoted by $d_{f}$; it is given by $d_{f}(r)=m\left(f^{-1}(-\infty, r)\right)$. If $T$ is measure-preserving, then $f \circ T$ and $f$ have the same distribution functions. So if $T$ is measure-preserving and $f \circ T=h \circ f$, then $d_{f}=d_{h \circ f}$, that is, the change of variables given by $h$ in the range of $f$ leaves the distribution function of $f$ invariant.

The converse of this discussion is summarized in the next proposition.

Proposition 4.1. Let $f \in L_{1}(X)$ be a generator. Suppose $h: \mathbb{R} \rightarrow \mathbb{R}$ is a Borel equivalence such that $d_{h \circ f}=d_{f}$. Then there exists a measure-preserving Borel map $T$ from $X$ onto $X$ such that $h \circ f=f \circ T$ and if $G \in L_{1}(X)$ is written as $G=g \circ f$ then $G \circ T^{k}=g \circ h^{k} \circ f$ for any natural number $k$.

Proof. Besides the distribution function $d_{F}$ for some $F \in L_{1}(X)$, we will also be using the Lebesgue-Stieltjes measure $m_{F}$ that is determined by $d_{F}$ on $\mathbb{R}$.

We begin with a couple of observations. Take any measurable function $G$. Since $G$ is $\Lambda$ measurable and $f$ is a generator, by Lemma 2.6, there exists a Borel measurable function $g: \mathbb{R} \rightarrow \mathbb{R}$ such that $G=g \circ f$.

The condition $d_{h \circ f}=d_{f}$ means that

$$
m\left(f^{-1}(-\infty, \alpha)\right)=m\left((h \circ f)^{-1}(-\infty, \alpha)\right)
$$

for a real number $\alpha$, which implies that

$$
m\left(f^{-1}(E)\right)=m\left(f^{-1} h^{-1}(E)\right)
$$

for every Borel set $E$ in $\mathbb{R}$. That is, $m_{f}=m_{h \circ f}$.

Now in particular, if $G=g \circ f$ as above, then $g^{-1}(-\infty, \alpha)$ is a Borel measurable set and

$$
\begin{aligned}
d_{g \circ h \circ f}(\alpha) & =m\left(f^{-1} h^{-1} g^{-1}(-\infty, \alpha)\right) \\
& =m\left(f^{-1} g^{-1}(-\infty, \alpha)\right) \\
& =m\left(G^{-1}(-\infty, \alpha)\right)=d_{G}(\alpha) .
\end{aligned}
$$

Therefore, the distribution functions $d_{g \circ h \circ f}$ and $d_{G}$ are equal. Hence, the measures $m_{g \circ f}$ and $m_{g \circ h \circ f}$ on $\mathbb{R}$ are the same.

Let $\mathscr{L}_{1}(X)$ be the integrable Lebesgue measurable functions, not identified a.e. as with $L_{1}(X)$. Consider a map $U$ on $\mathscr{L}_{1}(X)$, taking values in the measurable functions 
on $X$, defined by $U(G)=g \circ h \circ f$ whenever $G=g \circ f$ as above. It is clear that if $U$ is well-defined then it is a linear map. Indeed, if $G_{i}=g_{i} \circ f, i=1,2, a$ is a constant then $a G_{1}+G_{2}=\left(a g_{1}+g_{2}\right) \circ f$ and $U\left(a G_{1}+G_{2}\right)=\left(a g_{1}+g_{2}\right) \circ h \circ f=a g_{1} \circ h \circ f+g_{2} \circ h \circ f=$ $a U\left(G_{1}\right)+U\left(G_{2}\right)$.

To see that $U$ is actually well-defined, notice that a measurable function $\phi=0$ a.e. if and only if $d_{\phi}(\alpha)$ is a step-function which is 0 for $\alpha \leq 0$ and 1 for $\alpha>0$ (i.e., $d_{\phi}$ is the Heaviside function). Now suppose that a function $G \in \mathscr{L}_{1}(X)$ is 0 a.e., and write $G=$ $g \circ f$. Then the equality $d_{g \circ h \circ f}=d_{G}$ implies that $g \circ h \circ f=0$ a.e. on $X$. Hence, we have $U$ being a well-defined linear mapping on $L_{1}(X)$. Since the measures $m_{g \circ f}$ and $m_{g \circ h \circ f}$ are equal,

$$
\begin{aligned}
\int_{X}|G(x)| d m(x) & =\int_{\mathbb{R}}|\alpha| d m_{g \circ f}(\alpha) \\
& =\int_{\mathbb{R}}|\alpha| d m_{g \circ h \circ f}(\alpha) \\
& =\int_{X}|g \circ h \circ f| d m(x) .
\end{aligned}
$$

Hence, we see that actually $U: L_{1}(X) \rightarrow L_{1}(X)$ and $U$ an isometry.

By Lamperti's theorem (see Royden [3, page 416]) there exists a Borel measurable map $T$ from $X$ onto $X$ and a $K \in L_{1}(X)$ such that

$$
U(G)=K \cdot(G \circ T)
$$

From the proof of Lamperti's theorem, $K=U(1)$. Writing $1=g \circ f$ we see that $g=1$ and then $K=U(1)=g \circ h \circ f=1$. Therefore, whenever $G \in L_{1}(X)$ is written as $G=g \circ f$ we have

$$
G \circ T=g \circ h \circ f
$$

and for $f=i d \circ f$ we have

$$
f \circ T=h \circ f
$$

It is easy to show by induction that for $G=g \circ f$ and any natural $k$

$$
G \circ T^{k}=g \circ h^{k} \circ f \text {. }
$$

Finally, because $U$ is an isometry, the mapping $T$ is measure-preserving.

Remark 4.2. There is an alternative way to show that the map $U$ in the previous proof is well defined. Notice first that if $G=g \circ f \geq 0$ a.e. then also $g \circ h \circ f \geq 0$ a.e. This follows from the fact that $d_{g \circ h \circ f}=d_{G}$. Now suppose that $G_{1}=G_{2}$ a.e., where $G_{1}=g_{1} \circ f, G_{2}=$ $g_{2} \circ f$. Then $G_{1}-G_{2}=\left(g_{1}-g_{2}\right) \circ f \geq 0$ a.e. implies $\left(g_{1}-g_{2}\right) \circ h \circ f \geq 0$, that is, $g_{1} \circ h \circ$ $f \geq g_{2} \circ h \circ f$ a.e. Since also $G_{2}-G_{1} \geq 0$ a.e., we actually have $g_{1} \circ h \circ f=g_{2} \circ h \circ f$ a.e. Therefore $U(G)=g \circ h \circ f$ where $G=g \circ f$ is well defined. 


\section{Measurable and quantitative estimation}

5.1. Measurable estimation. Suppose that we are given $\left(\mathscr{B}_{n}\right)$, a non-decreasing sequence of sub- $\sigma$-algebras of $\Lambda$. These could be determined by a sequence $\left(g_{n}\right)$ of measurable functions in the sense that $\mathscr{B}_{n}=\sigma\left(g_{1}, \ldots, g_{n}\right)$ for every $n \geq 1$. But by Corollary 2.4 and Lemma 2.6, this is the same as having sequences $\left(h_{n}\right)$ and $\left(\Delta_{n}\right)$ such that $\mathscr{B}_{n}=\sigma\left(h_{n}\right)$ and $h_{n}=\Delta_{n} \circ h_{n+1}$ for all $n \geq 1$. Given this setup, we would like to be able to recognize when, for some $f \in L_{1}(X)$, the sequence $\left(f \circ T^{n}\right)$ is such that $\sigma\left(f \circ T^{n}\right) \subset \sigma\left(h_{n}\right)$ for all $n \geq 1$. In this situation, we say that $\left(h_{n}\right)$ is a measurable estimator for $(f, T)$.

Remark 5.1. Most of the time, a fixed sequence $\left(h_{n}\right)$, with $\sigma\left(h_{n}\right) \subset \sigma\left(h_{n+1}\right)$ for all $n \geq 1$, will be a measurable estimator for all $f$. This is because generically $h_{1}$, and hence any of the other $h_{n}$, is a generator. But also, generally a non-trivial fixed sequence $\left(h_{n}\right)$ will not be a measurable estimator for any $f$, indeed $\sigma\left(f \circ T^{n}\right)$ is not a subset of $\sigma\left(h_{n}\right)$ for any $n \geq 1$. This is because a non-trivial fixed sequence would have $\sigma\left(h_{n}\right) \neq \Lambda$, while the generic function $f$ is a generator, and so all the iterates $f \circ T^{n}$ will be generators too.

How would we recognize when we have a measurable estimator $\left(h_{n}\right)$ for some $(f, T)$ without knowing $(f, T)$ explicitly? We can get a good characterization in terms of generators.

Proposition 5.2. Fix a generator $f_{0}$. A sequence of $\Lambda$-measurable functions $\left(h_{n}\right)$ is a measurable estimator for some $(f, T)$ if and only if there exist Borel measurable functions $F,\left(\Delta_{n}\right.$ : $n \geq 1),\left(H_{n}: n \geq 1\right)$, and $\left(s_{n}: n \geq 0\right)$ such that

(a) $h_{n}=\Delta_{n} \circ h_{n+1}$ for $n=1,2,3, \ldots$,

(b) $h_{n}=H_{n} \circ f_{o}$ for $n=1,2,3, \ldots$, and

(c) $s_{n} \circ H_{n} \circ f_{o}=F \circ s_{o}^{n} \circ f_{o}$ for $n=1,2,3, \ldots$

(d) The distribution functions of $f_{o}$ and $s_{o} \circ f_{o}$ are equal.

Proof. Here is how the conditions (a), (b), (c), and (d) arise. Assume that $\left(h_{n}\right)$ is a measurable estimator for the pair $(f, T)$. Then by Lemma 2.6, since $\sigma\left(h_{n}\right) \subset \sigma\left(h_{n+1}\right),\left(\Delta_{n}\right)$ exists and so (a) holds. Then, using the same lemma, we can choose Borel measurable functions $\left(H_{n}\right)$ such that (b) holds. But then since we are assuming that $\sigma\left(f \circ T^{n}\right) \subset \sigma\left(h_{n}\right)$, it also follows that there exist Borel measurable functions $\left(s_{n}: n=0,1,2,3, \ldots\right)$ such that $s_{n} \circ h_{n}=s_{n} \circ H_{n} \circ f_{o}=f \circ T^{n}$ for all $n=1,2,3, \ldots$, and $s_{o} \circ f_{o}=f_{o} \circ T$. If we then take a Borel measurable function $F$ such that $f=F \circ f_{o}$, we would have $F \circ s_{o}^{n} \circ f_{o}=F \circ f_{o} \circ$ $T^{n}=f \circ T^{n}=s_{n} \circ H_{n} \circ f_{o}$ for all $n=1,2,3, \ldots$ This is (c). Moreover, (d) follows because $f_{o}$ and $f_{o} \circ T=s_{o} \circ f_{o}$ have the same distribution functions.

Conversely, the first assumption (a) guarantees that the $\sigma$-algebras $\sigma\left(h_{n}\right)$ are increasing. Then assumption (d) and Proposition 4.1, tells us that there is a measure-preserving transformation $T$ such that $s_{o} \circ f_{o}=f_{o} \circ T$. Then assumptions (b) and (c) tell us that with $f=F \circ f_{o}$, we have $\sigma\left(f \circ T^{n}\right) \subset \sigma\left(h_{n}\right)$ for all $n=1,2,3, \ldots$

In some special situations, we can have the interesting case that a function $f$ can end up being a measurable estimator for its own iterates $f \circ T^{n}$ for suitable powers $n \geq 1$. The extreme case of this is when $\sigma\left(f \circ T^{n+1}\right) \subset \sigma\left(f \circ T^{n}\right)$ for all $n \geq 0$. This is equivalent to having $\sigma\left(f \circ T^{n}\right) \subset \sigma(f)$ for all $n \geq 1$. Of course, this will happen if $f$ is a generator. 
A somewhat less restrictive condition is that this happens eventually, that is for some $N \geq 1$, if $n>N$, then $\sigma\left(f \circ T^{n}\right) \subset \sigma\left(f, f \circ T, \ldots, f \circ T^{N}\right)$. For example, this turns out to be a well-known phenomenon in the case of characteristic functions $f$. Then the $\sigma$ algebra $\sigma\left(f, \ldots, f \circ T^{n}\right)$ is always finite and this creates a special situation. We will say that $A$ is an eventual measurable estimator if for some $N \geq 1$, if $n>N$, then $\sigma\left(1_{A} \circ T^{n}\right) \subset$ $\sigma\left(1_{A}, 1_{A} \circ T, \ldots, 1_{A} \circ T^{N}\right)$.

Proposition 5.3. T is not totally ergodic if and only if some $A \in \Lambda$ with $0<m(A)<1$ is an eventual measurable estimator.

Proof. If $T$ is not totally ergodic, then $T^{n}$ is not ergodic for some $n$, and there exists a set $A \in \Lambda$ with $0<m(A)<1$ which is invariant under $T^{n}$. Then the sequence $1_{A}, 1_{A}$ 。 $T, 1_{A} \circ T^{2}, \ldots$ is periodic, and so clearly $A$ is an eventual estimator. For the other direction, the finiteness of $\sigma\left(1_{A}, \ldots, 1_{A} \circ T^{n}\right)$ and $A$ being an eventual estimator allows one to use the Pigeon-Hole Principle to see that there exists some $m \geq 1$ and $k>m$ such that $1_{A} \circ T^{k}=1_{A} \circ T^{m}$. So with $r=k-m$, we get $T^{r}$ leaving $A$ invariant and so $T^{r}$ is not ergodic.

It follows from Proposition 5.3 that if $T$ is weakly mixing, or just has no eigenvalues that are roots of unity, then any non-trivial measurable set $A$ is not an eventual estimator. Indeed, in any case we have the following generic situation.

Proposition 5.4. If $T$ is ergodic, then the sets $A \in \Lambda$ that are not an eventual estimator form a dense $G_{\delta}$ subset of $(\Lambda /$ a.e., $\rho)$.

Proof. Consider the collection $P$ of sets $A \in \Lambda$ /a.e. such that there exists some $n>1$ with $T^{n} A=A$ a.e. We only need to show that this is a countable union of closed sets with no interior. Indeed, $P$ is the countable union of sets $P_{n}=\left\{A \in \Lambda: T^{n} A=A\right.$ a.e. $\}$, where $n>$ 1. It is easy to see that each of these sets is closed in the $\rho$-topology. But these sets also have no interior. Indeed, suppose $A \in P_{n}$ and let $\epsilon>0$. Assume for the moment that $m\left(A^{c}\right)>0$. The Rokhlin lemma tells us that for any $\epsilon>0$ and $M \geq 1$, there is a measurable set $B$ such that $B, T B, \ldots, T^{M} B$ are pairwise disjoint and $m\left(X \backslash \bigcup_{k=0}^{M} T^{k} B\right)<\epsilon$. But then since $m\left(A^{c}\right)>0$, we can use the Rokhlin lemma to see that there is a choice of $M$ such that the measurable set $B$ in the Rokhlin lemma has $0<m(B)<\epsilon$ and for some $k \geq 1$ with $k+n \leq$ $M$, we also know that $m\left(A^{c} \cap T^{k} B\right)>0$. Consider the set $C=A \cup T^{k} B$. Then $\rho(A, C)<\epsilon$. We claim that $C \notin P_{n}$. If it were, then $T^{n} C=A \cup T^{n+k} B=C=A \cup T^{k} B$ a.e. Since $T^{k} B$ and $T^{n+k} B$ are disjoint, this means that $T^{k} B \subset A$ a.e. which contradicts $m\left(A^{c} \cap T^{k} B\right)>0$. On the other hand, if $m\left(A^{c}\right)=0$, then use the Rokhlin lemma to find a set $B$ with $0<m(B)<\epsilon$ and $B$ and $T^{n} B$ are disjoint. Let $C=A \backslash B$. Then $\rho(A, C)<\epsilon$. Also, $C \notin P_{n}$ since if it were then $T^{n} C=A \backslash T^{n} B=C=A \backslash B$ which implies that $T^{n} B=\left(A \backslash T^{n} B\right)^{c}=(A \backslash B)^{c}=B$ contradicting the disjointness of $B$ and $T^{n} B$. In any case, since $\epsilon>0$ was arbitrary, no set $A \in P_{n}$ can be an interior point of $P_{n}$.

The conclusion of the discussion above is that the concept of being an eventual estimator does not apply to the generic characteristic function in an ergodic dynamical system. As we have seen, when we showed that the generic function is a generator, the situation is quite different when one considers the general function $f \in L_{1}(X)$. 
5.2. Quantitative estimation. We now discuss another possibility: quantitative estimation from the ergodic averages. As a general principle, we should be able to make estimations if we have sufficient information. For example, if we know that a particular function $f \in L_{1}(X)$ has been chosen and we know that for very large values of $n$, $A_{n} f=(1 / n) \sum_{k=0}^{n-1} f \circ T^{k}$ is very close to 0 with high probability, then we should be able to say that $\int_{X} f d m=0$. This is an abstraction of the basic principle in any quantitative form of the law of large numbers. However, we also know that there is no rate of convergence in the ergodic theorem; see for example, del Junco and Rosenblatt [1] and Kakutani and Petersen [2]. Consequently, we do not know how large $n$ must be, that is, how long we have to wait, for our estimates to be reasonably accurate without having more information about the function $f$.

We could also try to gain more information about the function than just its mean value from behavior of the ergodic averages. This is a more complicated issue that we discuss some at the end of this section. For now, let us focus on estimating facts about the mean of the function from information about the ergodic averages.

We can see that sometimes we can make estimates based on knowledge of some of the ergodic averages. However, sometimes we cannot. The fact that this is connected with which $L_{p}$-space we are in or whether the function is positive valued, makes this dichotomy even more interesting. Here is a positive result that allows us to estimate the integral of a function, and see that it is small, given information about some of the ergodic averages.

Proposition 5.5. Suppose $\epsilon>0, \delta<1$, and $K<\infty$. Suppose that we have some function $f \in L_{p}(X), 1<p<\infty$, with $\|f\|_{p} \leq K$, and we observe that for some $N$, with probability at least $\delta$, we have $\left|A_{N} f(x)\right| \leq \epsilon$. Then, $\left|\int_{X} f d m\right| \leq \epsilon+K(1-\delta)^{(p-1) / p}$.

Proof. Let $E=\left\{x \in X:\left|A_{N} f(x)\right| \leq \epsilon\right\}$. Let $q$ be the index conjugate to $p$ so that $(p-1) / p=1 / q$. Of course, we always have $\int_{X} f d m=\int_{X} A_{N} f d m$. So using Hölder's Inequality, we have

$$
\begin{aligned}
\left|\int_{X} f d m\right| & \leq \int_{E}\left|A_{N} f\right| d m+\int_{E^{c}}\left|A_{N} f\right| d m \\
& \leq \epsilon m(E)+m\left(E^{c}\right)^{1 / q}\|f\|_{p} \\
& \leq \epsilon+\|f\|_{p}(1-\delta)^{1 / q} .
\end{aligned}
$$

Since we are assuming $\|f\|_{p} \leq K$, this gives the inequality that we wanted.

This proposition shows that one can give a quantitative estimate that guarantees that $\int_{X} f d m$ is not large without knowing anything explicit about $f$ except that most of the values of $\left|A_{N} f(x)\right|$ are small for some value of $N$. We do need to assume though that there is some global control on the $L_{p}$-norm of the function. But we also needed to assume that we had a function $f \in L_{p}(X)$ with $p>1$. The next proposition shows that this ability to make an estimation breaks down when $p=1$.

We will formalize this as follows. Consider the domain where $\epsilon>0, \delta<1$, and $M \leq N$ such that also $\delta \leq m\left\{x \in X: \sup _{M \leq n \leq N}\left|A_{n} f(x)\right| \leq \epsilon\right\}$. Assume that we have a function $F(\epsilon, \delta, M, N)$ defined on this domain that tends to 0 when we have jointly $\epsilon$ tending to 0 , $\delta$ tending to 1 , and $M$ tending to $\infty$. We call $F$ a quantitative over estimator if $\left|\int_{X} f d m\right| \leq$ $F(\epsilon, \delta, M, N)$. Part of the reason that we consider such an over estimator is that if we 
fix $f \in L_{1}(X)$, and we knew that $\int_{X} f d m=0$, then for all $\delta<1$ and $\epsilon>0$, we can find large enough $M$ so that for all $N$, we have $\delta \leq m\left\{x \in X: \sup _{M \leq n \leq N}\left|A_{n} f(x)\right| \leq \epsilon\right\}$. So we might anticipate conversely that if this condition held, then we could find a function $F(\epsilon, \delta, M, N)$ to use in making the quantitative estimate $\left|\int_{X} f d m\right| \leq F(\epsilon, \delta, M, N)$ that $\int_{X} f d m$ is small for suitable choices of $\epsilon, \delta, M$, and $N$.

If we restrict our function to be in $L_{p}(X)$, then $F(\epsilon, \delta, M, N)=\epsilon+\|f\|_{p}(1-\delta)^{(p-1) / p}$ is a quantitative over estimator given the appropriate domain restriction. Indeed, the domain restriction $\delta \leq m\left\{x \in X: \sup _{M \leq n \leq N}\left|A_{n} f(x)\right| \leq \epsilon\right\}$ implies that $\delta \leq m\{x \in X$ : $\left.\left|A_{N} f(x)\right| \leq \epsilon\right\}$. So Proposition 5.5 is proving that this $F$ is a quantitative over estimator. Actually, Proposition 5.5 is saying that if we replace $\|f\|_{p}$ by $K$, then we would have a quantitative over estimator that is effective uniformly on the ball of radius $K$ in $L_{p}(X)$.

These introductory remarks should make clear the role of the following result.

Proposition 5.6. Given an ergodic transformation, there is no quantitative over estimator in $L_{1}(X)$.

Proof. Suppose we have a quantitative over estimator $F(\epsilon, \delta, M, N)$ for $L_{1}(X)$. Fix the values of $\epsilon, \delta, M, N$ so that $F(\epsilon, \delta, M, N) \leq 1 / 2$. For $L$ much larger than $N$ and $\gamma$ much smaller than $1-\delta$, choose a Rokhlin stack $S$ of height $L$ such that $m(X \backslash S) \leq \gamma$. Fix $K$ large and let $D$ be a subset of the base $B$ of the stack of measure $1 / K L$. Let $f$ be the simple function with value $K$ on all of the images $D, T D, \ldots, T^{L-1} D$ and 0 otherwise. Then $f$ is positive and $\int_{X} f d m=\|f\|_{1}=1$. However, for a suitable choice of $K$, $L$, and $\gamma$, the averages $A_{n} f, M \leq n \leq N$ are all so close to $f$ in norm that we also have $\delta \leq m\left\{x \in X: \sup _{M \leq n \leq N} A_{n} f=0\right\} \leq m\left\{x \in X: \sup _{M \leq n \leq N}\left|A_{n} f\right| \leq \epsilon\right\}$. Since $F$ is a quantitative over estimator, we would have $1=\int f d m \leq F(\epsilon, \delta, M, N) \leq 1 / 2$. This contradiction shows there cannot be a quantitative over estimator in $L_{1}(X)$.

Remark 5.7. The proof of Proposition 5.6 shows that there cannot even be a quantitative over estimator in $L_{1}(X)$ when one restricts the norm of the function to keep it inside a predetermined ball in $L_{1}(X)$.

We could have shifted our viewpoint above to one where we would use information about the averages $A_{n} f$ to estimate that $\int_{X} f d m$ is large, instead of estimating that it is small. When we do this, the role of the $L_{p}$-space in the problem shifts so that $L_{1}(X)$ becomes a good space to work in, at least for positive functions. Here is an obvious very simple argument that gives an example of a lower estimate that is possible in $L_{1}(X)$.

Proposition 5.8. Suppose we have a positive function $f \in L_{1}(X)$. Fix $s>0$ and $\delta<1$. Suppose that we have values of $M$ and $N$ so that with probability at least $\delta$, we have $\sup _{M \leq n \leq N}\left|A_{n} f\right| \geq s$. Then we have $\int_{X} f d m \geq s \delta$.

Proof. Since the function is positive, this proposition follows immediately from the weak $(1,1)$ maximal estimate in ergodic theory:

$$
m\left\{x \in X: \sup _{n \geq 1}\left|A_{n} f\right| \geq s\right\} \leq \frac{\|f\|_{1}}{s} .
$$


Remark 5.9. The underestimate in this proposition gives us a significant underestimate only if $s \delta$ is large. So even if $\delta$ is small, as long as $s$ is sufficiently large, we will have such a significant underestimate. Also, the same result is of course true using all values of $n \leq N$. We have stated the result this way to emphasize that it does not matter that one does not have data about the averages $A_{n} f$ for small values of $n$ in order to get an underestimate of the mean of $f$.

However, the underestimate in Proposition 5.8 will not work in any manner if we do not assume the function $f$ is positive. We can quantify this as follows. Consider the domain where $s>0, \delta<1$, and $N \geq 1$ such that also $\delta \leq m\left\{x \in X: \sup _{n \leq N}\left|A_{n} f\right| \geq s\right\}$. Assume that we have a function $F(s, \delta, N)$ defined on this domain with the property: there is a constant $c>0$ such that for large enough $s$ and $N$, and for $\delta$ close enough to 1 , we have $F(s, \delta, N) \geq c$. Under these conditions, if we always have $F(s, \delta, N) \leq\left|\int_{X} f d m\right|$, then we say that $F$ is a quantitative under estimator. Clearly, this is the concept analogous to over estimation with the inequality in the reverse direction. If we restrict our functions to being positive, then $F(s, \delta, N)=s \delta$ is a quantitative under estimator. Indeed, this is exactly the content of Proposition 5.8.

Proposition 5.10. Given an ergodic transformation, there is no quantitative under estimator in $L_{r}(X), r \geq 1$ unless the function is positive.

Proof. Since $F$ is a quantitative under estimator, we can choose $s, \delta, N$ such that $\delta \leq$ $m\left\{x \in X: \sup _{n \leq N}\left|A_{n} f\right| \geq s\right\}$ and $F(s, \delta, N) \leq\left|\int_{X} f d m\right|$. There is no harm in assuming that $s$ and $N$ are sufficiently large, and $\delta$ is sufficiently close to 1 , such that $F(s, \delta, N) \geq$ $c / 2$. For $L$ much larger than $N$ and $\gamma$ much smaller than $1-\delta$, choose a Rokhlin stack $S$ of height $L$ such that $m(X \backslash S) \leq \gamma$. Let $D$ be a subset of the base $B$ of the stack of measure $(1 / 2) m(B)$. Let $f$ be the simple function with non-zero values on $S$, and values 0 otherwise, such that $f=2 s$ on all of the images $D, T D, \ldots, T^{L-1} D$ and $f=-2 s$ on all the images $D^{c}, T D^{c}, \ldots, T^{L-1} D^{c}$. Then $|f|=2 s$ on $S$ and $\int_{X} f d m=0$. However, for a suitable choice of $L$ and $\gamma$, the averages $A_{n} f, n \leq N$, are all so close to $f$ in $L_{1}$-norm that we have $\delta \leq m\left\{x \in X: \sup _{n \leq N}\left|A_{n} f\right| \geq s\right\}$. Since $F$ is a quantitative under estimator, we have $c / 2 \leq F(s, \delta, N) \leq \int f d m=0$. This contradiction shows there cannot be a quantitative under estimator in $L_{1}(X)$. If $f$ is positive then there is a quantitative under estimator. Use Proposition 5.8 and the fact that $L_{r}(X) \subset L_{1}(X)$. Notice though that because of the disparity of norms, we would typically have $\left|\int_{X} f d m\right|$ being much smaller than $\|f\|_{p}=1$.

We see in this construction that $\|f\|_{\infty}=2 s$ on most of the space. So in order to show there is no quantitative under estimator as we have stated the definition, we need to use functions $f \in L_{1}(X)$ of potentially very large $L_{1}$-norm. We could fix this by requiring only that $s$ be chosen close enough to a bound $K$, with $N$ very large and $\delta$ close to 1 , to get the underestimate $c / 2 \leq F(s, \delta, N)$. Then the functions in the construction can be kept inside a ball of radius $K$ in $L_{1}(X)$. This would be a reasonable adjustment in the discussion above, if we are to be dealing with a good quantitative under estimator, since then we should be assuming that the terms $c$, s, and $\left|\int f d m\right|$ are all essentially the same size. 
We can use the ideas of quantitative estimators to gain more information about a function $f \in L_{1}(X)$ than just its mean. Here is an example of the general idea. Recall that our measure space is $([0,1], \Lambda, m)$, with $\Lambda$ being the Lebesgue measurable sets and $m$ being Lebesgue measure. Let $T$ be an ergodic transformation. For each $y \in X$, consider the averages $A_{n}\left(f 1_{[y, y+\epsilon]}\right)$. If we could use data from the averages $A_{n}\left(f 1_{[y, y+\epsilon]}\right)$ to estimate mean values, then we could compute $\int_{y}^{y+\epsilon} f(x) d s$ for various values of $y$ and $\epsilon$. Dividing these by $\epsilon$, we could estimate values of $f(y)$ for a.e. $y$. In this manner, we could recover the function $f$ from data about ergodic averages. The method that we would use here is that we would first have a good way of observing $T^{k}(x)$ so that we would be sampling $f\left(T^{k} x\right)$ only when $T^{k}(x) \in[y, y+\epsilon]$. This adds serious inaccuracies and difficulties to any program of data sampling in ergodic theory.

\section{References}

[1] A. del Junco and J. Rosenblatt, "Counterexamples in ergodic theory and number theory," Mathematische Annalen, vol. 245, no. 3, pp. 185-197, 1979.

[2] S. Kakutani and K. Petersen, "The speed of convergence in the ergodic theorem," Monatshefte für Mathematik, vol. 91, no. 1, pp. 11-18, 1981.

[3] H. L. Royden, Real Analysis, Macmillan, New York, NY, USA, 3rd edition, 1988.

Svetlana Butler: Department of Mathematics, University of Illinois at Urbana, Urbana, IL 61801, USA

E-mail address: svbutler@math.uiuc.edu

Joseph Rosenblatt: Department of Mathematics, University of Illinois at Urbana, Urbana, IL 61801, USA

E-mail address: jrsnbltt@math.uiuc.edu 


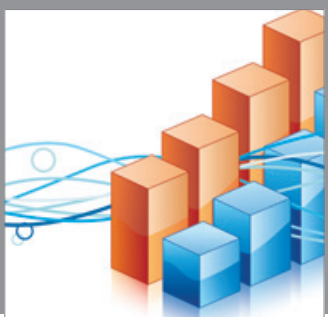

Advances in

Operations Research

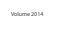

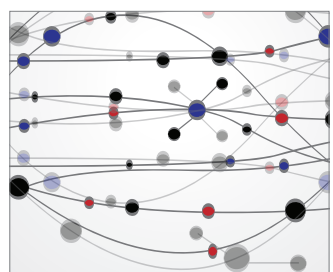

\section{The Scientific} World Journal
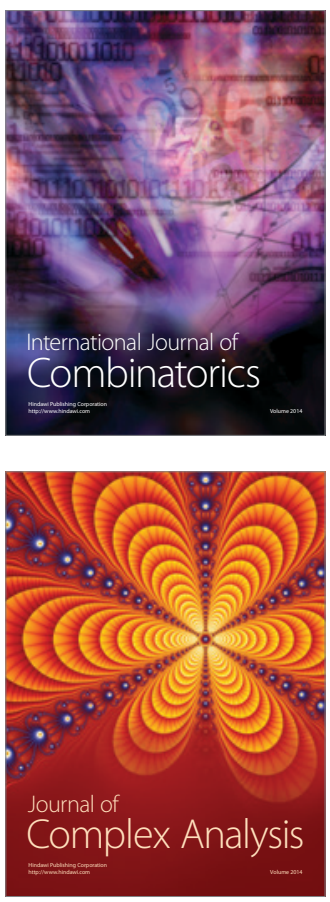

International Journal of

Mathematics and

Mathematical

Sciences
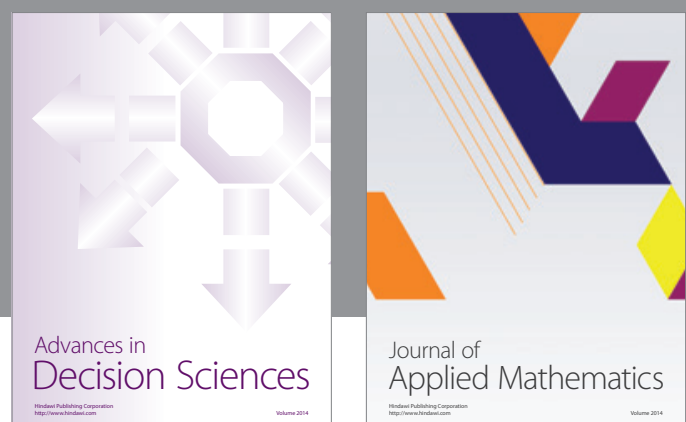

Journal of

Applied Mathematics
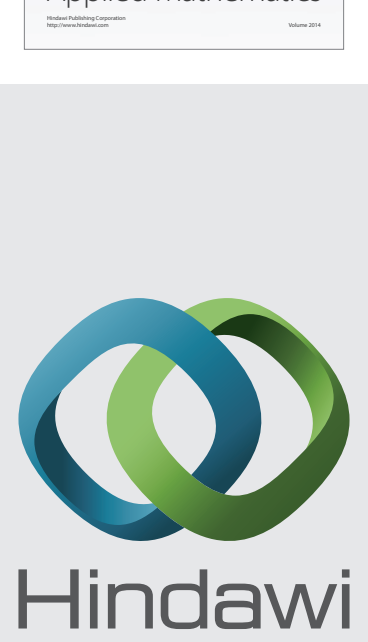

Submit your manuscripts at http://www.hindawi.com
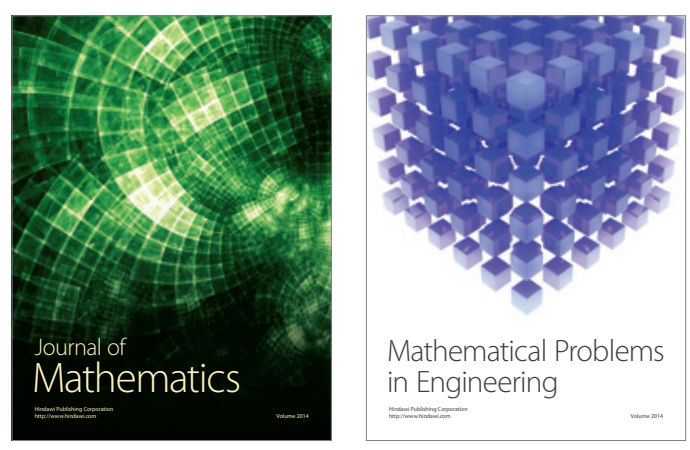

Mathematical Problems in Engineering
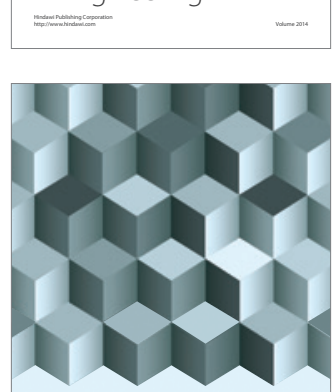

Journal of

Function Spaces
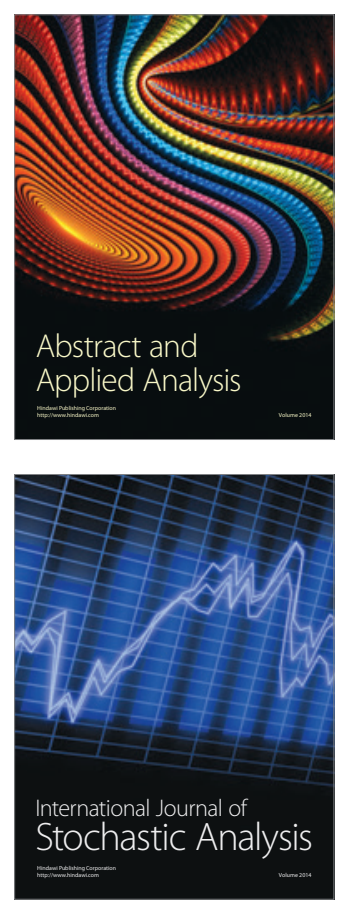

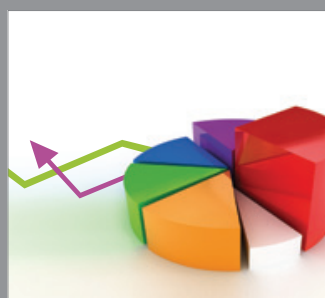

ournal of

Probability and Statistics

Promensencen
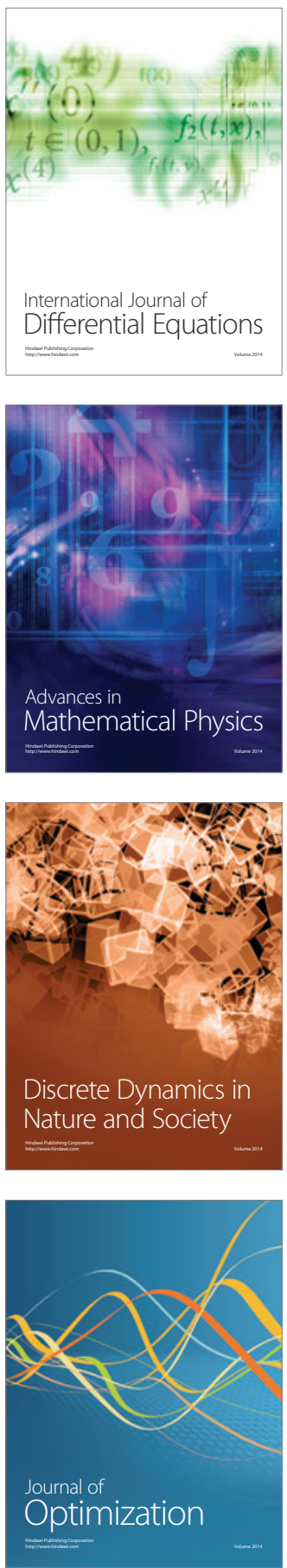